\title{
Three-dimensional bifurcations of a two-phase Rayleigh-Benard problem in a cylinder
}

\author{
C.W. Lan *, C.H. Wang \\ Department of Chemical Engineering, National Taiwan University, Taipei 10617, Taiwan, ROC
}

Received 3 March 2000; received in revised form 26 July 2000

\begin{abstract}
Three-dimensional (3D) bifurcations of a partially melted or solidified material in a cylinder heated from below are studied numerically. Through nonlinear calculations, bifurcation diagrams are constructed for a melt of a Prandtl number of one. As the interface is fixed, our calculated results agree reasonably well with previous calculations, but some discrepancies exist, which are further discussed through their dynamic evolutions and imperfect bifurcations of $5^{\circ}$ tilt. As the interface is allowed to deform, the bifurcation behavior changes significantly, both for the onset of convection and its convection mode. For the initial melt aspect ratio of one, the primary bifurcation changes from supercritical to subcritical with the increasing solid amount, and the onset mode from an axisymmetric $(m 0)$ mode to a 3D ( $m 1)$ mode. Although the free interface destabilizes the conductive mode and leads to an earlier onset of convection, it may stabilize some flow modes through its confinement. Imperfect bifurcations due to a $5^{\circ}$ tilt are further illustrated. (c) 2001 Elsevier Science Ltd. All rights reserved.
\end{abstract}

Keywords: Three-dimensional; Bifurcation; Rayleigh-Benard; Buoyancy flow; Interface

\section{Introduction}

In many physical problems in nature or industrial processes, thermal convection is an important mechanism for heat and mass transfer. Particularly, as a fluid is heated from below, its nonlinear behavior is very rich and interesting in many scientific fields. The classic Rayleigh-Benard (RB) problem offers a first approach to complex flows as well as the transition from conductive to convective heat transfer modes. In many physical processes, the RB problem may further be associated with freezing or melting, where the onset of thermal convection and the flow modes are coupled with the deformable melt/solid interface. Such is the case for the storage of energy using the phase change of material $[1,2]$. In the solidification processing of alloys and the growth of single crystals [3-5], it is also particularly important. For example, during crystal growth of elec-

\footnotetext{
${ }^{*}$ Corresponding author. Tel.: +886-2-2363-3917; fax: +886-22363-3917.

E-mail address: lan@ruby.che.ntu.edu.tw (C.W. Lan).
}

tronic materials, the onset mode could affect the interface morphology and dopant segregation, which are crucial to crystal quality [3].

In engineering applications, the material or fluid is usually confined in a container, e.g., a box or a cylinder. A generic situation in a cylinder is shown in Fig. 1(a), where the fluid is heated from below. Because of the friction from the solid wall, the onset of convection and the flow bifurcations depend on the boundary conditions. Indeed, as compared with the buoyancy convection between two parallel plates of infinite extent, the bounded configuration has received much less attention because of the difficulty of its analytical and numerical treatment. In fact, in many engineering applications, such as crystal growth or solidification in an ampoule, this configuration is important. For this problem with only the fluid inside, its bifurcations (primary) have been well studied for the axisymmetric modes since the work by Liang et al. [6] and Charlson and Sani [7,8]. Detailed bifurcation diagrams were established by Yamaguchi et al. [9] through a fully nonlinear finite element analysis. Due to the limitation of the computation resources, its three-dimensional (3D) bifurcations were not 


\begin{tabular}{|c|c|c|c|}
\hline \multicolumn{2}{|c|}{ Nomenclature } & $T_{\mathrm{m}}$ & melting point \\
\hline$A$ & melt aspect ratio & $v$ & $x^{2}$-component of velocity \\
\hline A & surface area vector in Fig. 2(b). & $\Delta V$ & control volume \\
\hline$C_{\mathrm{p}}$ & specific heat & $\mathbf{v}$ & velocity vector \\
\hline $\mathbf{e}_{1}$ & unit vector in $x^{1}$-direction & $w$ & $x^{3}$-component of velocity \\
\hline $\mathbf{e}_{\mathrm{g}}$ & unit vector in the gravity direction & $\mathbf{x}$ & variable vector \\
\hline f & residual vector & $\mathbf{y}$ & variable vector $(\boldsymbol{x}, R a)^{\mathrm{T}}$ \\
\hline $\begin{array}{l}g \\
h\end{array}$ & $\begin{array}{l}\text { gravitational acceleration } \\
\text { melt height }\end{array}$ & \multicolumn{2}{|c|}{ Greek symbols } \\
\hline$\Delta H$ & heat of fusion & $\alpha_{\mathrm{m}}$ & thermal diffusivity of melt \\
\hline$H$ & domain height & $\beta_{\mathrm{T}}$ & thermal expansion coefficient \\
\hline I & fluxes & $\xi^{l}$ & coordinate in computational domain \\
\hline$k$ & thermal conductivity & $\gamma$ & tilt angle \\
\hline$L$ & length & $\kappa$ & thermal conductivity ratio $\left(k / k_{\mathrm{m}}\right)$ \\
\hline $\mathbf{n}$ & unit normal vector & $\theta$ & dimensionless temperature \\
\hline $\mathrm{Nu}$ & Nusselt number & $\tau$ & dimensionless time \\
\hline$P$ & pressure & $\tau_{i j}$ & singularity test function \\
\hline $\operatorname{Pr}$ & Prandtl number, $v_{\mathrm{m}} / \alpha_{\mathrm{m}}$ & $v_{\mathrm{m}}$ & kinematic viscosity \\
\hline$R$ & radius & \multicolumn{2}{|c|}{ Superscripts } \\
\hline$R a$ & thermal Rayleigh number, $g \beta_{\mathrm{T}} R^{3}\left(T_{\mathrm{H}}-T_{\mathrm{m}}\right) /$ & 1 & $x$-direction in the Cartesian coordinate \\
\hline$s$ & $\begin{array}{l}v_{\mathrm{m}} \alpha_{\mathrm{m}} \\
\text { arclength }\end{array}$ & 2 & $y$-component in the Cartesian coordinate \\
\hline$S$ & surface area & 3 & $z$-component in the Cartesian coordinate \\
\hline$S_{\phi}$ & source term of variable $\phi$ & * & reduced value \\
\hline St & Stefan number, $\Delta H / C p_{\mathrm{m}}\left(T_{\mathrm{H}}-T_{\mathrm{m}}\right)$ & \multicolumn{2}{|c|}{ Subscripts } \\
\hline$T$ & temperature & $\mathrm{c}$ & critical value \\
\hline & cold zone temperature & $\mathrm{s}$ & solid \\
\hline$T_{\mathrm{H}}$ & hot zone temperature & $\mathrm{m}$ & melt \\
\hline
\end{tabular}

investigated until recently. Muller et al. [10] studied the flow structures in a cylinder for a liquid metal (Prandtl number $\operatorname{Pr}=0.02)$ and water $(P r=6.7)$ both experimentally and numerically, and found that for water, the axisymmetric mode can be stable up to $10 R a_{\mathrm{c}}$, where $R a_{\mathrm{c}}$ is the primary critical Rayleigh number. However, a secondary bifurcation was found at $R a=2800$ with the $3 \mathrm{D}$ solutions having an $m 2$ symmetry. A latter study by Neumann [11] also obtained similar results but with more careful numerical calculations, where the mesh effects were discussed. Interestingly, Hardin and Sani [12] used a weakly nonlinear analysis to show, differently, that the $m 2$ mode branched from the $\mathrm{m} 0$ mode supercritically at $R a_{\mathrm{c}}=2800$, and beyond this secondary bifurcation the $\mathrm{m} 0$ mode became unstable. Such a controversy was commented on by Wagner et al. [13] who showed that the appearance of the $m 2$ mode at $R a=2800$ was possibly due to the numerical error of a too-coarse grid used by Neumann [11]. Nevertheless, later Wanschura et al. [14] used a mixed finite-difference/ Chebyshev collocation method to study the same problem for the aspect ratio ranging from 0.9 to 1.56 and for $P r=0.02$ and 1 , and commented that while the $m 2$ mode might appear but suspected that there was a small range near $R a=2800$ where the $m 2$ mode was not stable. Despite the fact that the stability of the $m 0$ and $m 2$ modes are still unclear, the bifurcation structure proposed by Hardin and Sani [12] is perhaps the most complete to date for $\operatorname{Pr}=6.7$. It is all the same difficult to make any judgment about the stability of the modes because the supercritical bifurcations are not structurally stable. A little imperfection, such as tilt or heat loss, can remove these points. Furthermore, these points are highly sensitive to numerical accuracy. So far, the reported results are still not mesh independent. At least, no one has proven that. Therefore, in addition to the perfect bifurcations with the ideal boundary conditions, imperfect bifurcations due to symmetry breaking also provide useful information of which modes being more stable. However, to the best of our knowledge no such study has been conducted on this problem. The energy analysis [14] is also a useful way to investigate the stabilizing (or destabilizing) mechanisms. However, obtaining a basic state is a numerical problem. In addition to numerical accuracy, the branching structure is still difficult to find. Newton's method with a continuation technique [15-17] has been proven to be an efficient way for bifurcation analyses, but it is extremely costly for $3 \mathrm{D}$ problems. 
(a)

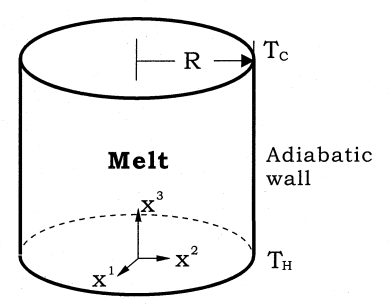

(b)

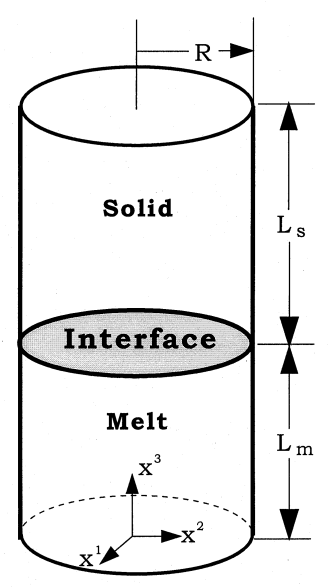

Conductive mode (c)

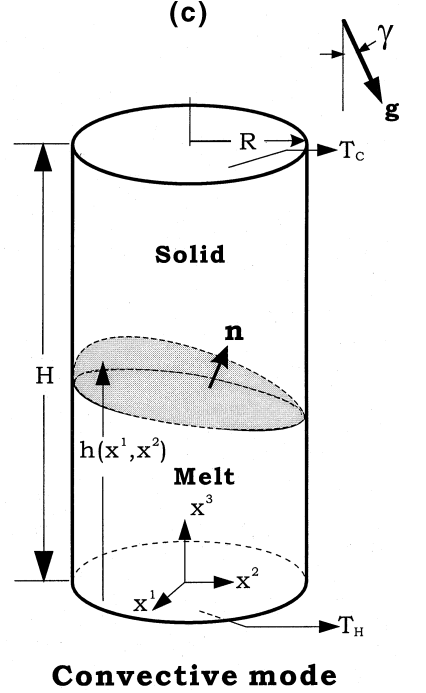

Fig. 1. Schematic of Rayleigh-Benard problems in a cylinder: (a) one-phase problem; (b) two-phase problem at a conductive mode; (c) two-phase problem at a convective mode.

As a materials is partially melted or solidified in a cylinder, as shown in Fig. 1(b), the bifurcation behavior for a two-phase RB problem could be quite different. After the onset of convection, the interface shape is a coupled solution with the bifurcated mode, as show in Fig. 1(c). Such a problem is particularly interesting and important to solidification and crystal growth, as well as in the applications toward energy storage problems using phase change materials. In fact, the coupled RB convection with solidification between two plates with infinitely extended domain was studied for the first time by Davis et al. [18]. They found that a subcritical bifurcation was associated with the onset of hexagonal cells for a thick solid layer. Limited by their weakly nonlinear analysis, their study was available only very near the static state. Through a fully nonlinear bifurcation and stability analyses, Lan et al. [15] constructed the bifurcation diagrams with detailed solution connectivity for a two-dimensional (2D) bounded case. Additional stable solution families and the transcritical bifurcation due to the interface concavity were found. For the cylinder configuration with an interface, Chang and Brown [16], for the first time, used a fully non- linear finite element analysis to construct its bifurcation diagrams. However, their calculations were based on the axisymmetric assumption, and the 3D bifurcations were not considered. They also found that the system became less stable, i.e., an earlier onset of convection, as the interface was introduced. The subcritical bifurcation due to the interface was not observed in their study.

From the previous 2D results [15], we expect that the coupling of the 3D bifurcations and solidification or melting could lead to different bifurcation behavior and thus different solution connections. If the solutions are structurally stable, these solutions will be of interest in practice, and they may have an impact on the design and operation. Further finding a subcriticality will be particularly useful because it causes a catastrophic change from a stable mode to the other. Therefore, in this paper we attempt to study, for the first time, the 3D bifurcations of the two-phase RB problem in a cylinder using a numerical bifurcation analysis. Through the fully nonlinear analysis, bifurcation diagrams can be constructed. From the diagrams, the onset of the flow and the possible (preferred) modes, as well as their linear 
stability, can be determined. The roles of the interface and the solid amount are further investigated.

In this paper, we only consider the material being pure or almost pure so that the solutal effects and the mushy zone can be neglected. In most energy storage or materials processing applications, e.g., the vertical Bridgman crystal growth [3], the material is confined in a container, i.e., with solid walls. Therefore, the use of the no-slip wall as the boundaries does not lose its generality. To generalize the system, the orientation (tilt angle $\gamma$ ) of the cylinder is arbitrary, so that the effect of tilting (imperfect bifurcation) can also be studied. For comparison, we will first construct the bifurcation diagram for the fixed-interface problem. As mentioned previously, the detailed stability of the convective modes is still controversial, and most of the arguments are due to numerical resolution. The $3 \mathrm{D}$ calculations of the bifurcation and stability analyses require tremendous computation effort, and it is still difficult to get meshindependent results. Therefore, in this paper, even though we also construct the bifurcation diagrams and propose some possible stable states for the fixed-interface problem, we do not attempt to settle the controversy. Instead, we are more interested in the solutions that appear to be more stable even under an imperfect condition. Furthermore, from there, we can investigate the role of the deformable interface on the bifurcation structures. Again, the preferred modes are proposed.

In the next section, the model description is presented, followed by numerical solutions in Section 3 . Section 4 is devoted to results and discussion. Conclusions and the comments are drawn in Section 5.

\section{Model description}

The schematic of the two-phase RB problem in an inclined cavity is shown in Fig. 1(c), which is represented in a Cartesian coordinate $\left(x^{1}, x^{2}, x^{3}\right)$. The height of the cylinder is $H$, which can be changed in this study, and the radius is $R$. The effects of melt aspect ratio $A$ is defined by $L_{\mathrm{m}} / R$, while the initial ratio of solid and melt heights is $L_{\mathrm{s}} / L_{\mathrm{m}}$. The temperature at the top and bottom ends is fixed; the lower temperature $T_{\mathrm{H}}$ is higher than the melting point $\left(T_{\mathrm{m}}\right)$ of the material inside, while the upper temperature $T_{\mathrm{C}}$ is lower. The side walls are assumed adiabatic; heat loss can be considered if necessary. The deformable melt/solid interface height $h\left(x^{1}, x^{2}\right)$ is unknown a priori and needs to be solved simultaneously with field variables. Physical properties of the material are assumed constant, while the melt is incompressible. Again, these assumptions can be relaxed if necessary.

Dimensionless variables are defined by scaling the length with $R$, velocity with $\alpha_{\mathrm{m}} / R$, and time with $R^{2} / \alpha_{\mathrm{m}}$, where $\alpha_{\mathrm{m}}$ is the thermal diffusivity. The dimensionless temperature $(\theta)$ is defined as $\theta \equiv\left(T-T_{\mathrm{m}}\right) /\left(T_{\mathrm{H}}-T_{\mathrm{m}}\right)$, while $\theta_{\mathrm{s}}$ is used for the solid phase. For the convenience of illustration, $T_{\mathrm{C}}$ is adjusted so that $\theta_{\mathrm{s}}$ is equal to -1 for $L_{\mathrm{s}} / L_{\mathrm{m}}=1$ at the upper boundary. The coordinates $\left(x^{1}, x^{2}, x^{3}\right)$, the interface height $h$, and velocity components $(u, v, w)$ used afterwards are all dimensionless. The Boussinesq approximation [19] is also adopted in the flow calculation. This assumption is reasonable if the temperature difference is not too large. Since the melt/ solid density ratio is usually about one for most materials, the volume change due to solidification or melting is neglected. The conservation equations in a dimensionless form for the 3D incompressible laminar flow of a Newtonian fluid and heat transfer in the melt can be described as follows:

$$
\begin{aligned}
& \nabla \cdot \mathbf{v}=0, \\
& \partial \mathbf{v} / \partial \tau+\mathbf{v} \cdot \nabla \mathbf{v}=-\nabla P+\operatorname{Pr} \nabla^{2} \mathbf{v}-\operatorname{Pr} \operatorname{Ra} \theta \mathbf{e}_{\mathrm{g}}, \\
& \partial \theta / \partial \tau+\mathbf{v} \cdot \nabla \theta=\nabla^{2} \theta,
\end{aligned}
$$

where $\tau, \mathbf{v}, P$, and $\theta$ are the dimensionless time, velocity, pressure, and temperature, respectively. $\operatorname{Pr}$ is the Prandtl number and $\mathbf{e}_{\mathrm{g}}$ the unit vector of gravity orientation. The associated dimensionless number $R a$ in the source term of the momentum equation is the thermal Rayleigh number, defined as follows:

$R a \equiv \frac{g \beta_{\mathrm{T}}\left(T_{\mathrm{H}}-T_{\mathrm{m}}\right) R^{3}}{\alpha_{\mathrm{m}} v_{\mathrm{m}}}$,

where $g$ is the gravitational acceleration, $\beta_{\mathrm{T}}$ the thermal expansion coefficients, and $v_{\mathrm{m}}$ is the kinematic viscosity.

In the solid $(s)$, only the conductive heat transfer needs to be considered

$\partial \theta_{\mathrm{s}} / \partial \tau=\kappa_{\mathrm{s}} \nabla^{2} \theta_{\mathrm{s}}$

where $\kappa_{\mathrm{s}} \equiv k_{\mathrm{s}} / k_{\mathrm{m}}$ is the dimensionless thermal conductivity of the solid, and it is assumed to be equal to one in this study.

The boundary conditions for temperature are set to be:

$\theta\left(x^{1}, x^{2}, 0\right)=1, \quad \theta_{\mathrm{s}}\left(x^{1}, x^{2}, H / R\right)=-1$,

for the bottom and top surfaces. The side wall is assumed adiabatic,

$\mathbf{n} \cdot \nabla \theta=0$,

where $\mathbf{n}$ is the normal vector of the solid wall pointing outward. For the velocity, the no-slip boundary condition is adopted

$\mathbf{v}=\mathbf{0}$.

More importantly, at the melt/solid interface, the interface energy balance is imposed as 
$S t\left(\mathbf{n} \cdot \mathbf{e}_{3}\right) \frac{\partial h}{\partial \tau}-\left.\kappa_{\mathrm{s}} \frac{\partial \theta_{\mathrm{s}}}{\partial n}\right|_{\mathrm{s}}+\left.\frac{\partial \theta}{\partial n}\right|_{\mathrm{m}}=0$,

as well as the melting-point isotherm:

$\theta=\theta_{\mathrm{s}}=0$.

In Eq. (8), $S t \equiv \Delta H /\left[C p_{\mathrm{m}}\left(T_{\mathrm{H}}-T_{\mathrm{m}}\right)\right]$ is the Stefan number, where $\Delta H$ is the heat of fusion and $C p_{\mathrm{m}}$ the specific heat of the melt. In Eq. (9), the effect of interfacial energy, i.e., the Gibbs-Thompson effect, is neglected because the system dimension is usually in the order of centimeters. This effect can be added easily if necessary. It should be pointed out that the numerical solution presented in this report can be used for a more general case. Therefore, the assumptions made so far are simply for the ease and clarity of illustration.

The measure of convective heat transfer is through the averaged Nusselt number $\overline{N u}$ at the melt/solid interface defined as

$\overline{N u}=-\frac{1}{S} \int_{S} \frac{\partial \theta}{\partial n} \mathrm{~d} S$

where $\mathbf{n}$ is the unit normal vector pointing toward the solid, and $S$ is the area of the interface. For the case without convection (pure heat conduction), $\overline{N u}=1$.

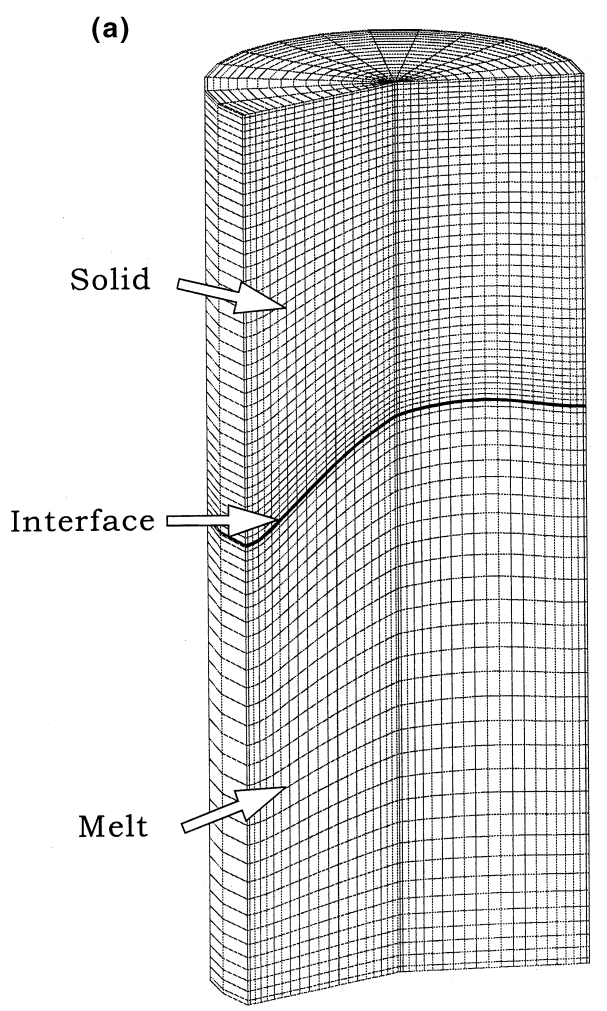

\section{Numerical solutions}

Due to the unknown and deformed interface shape $h\left(x^{1}, x^{2}\right)$, a finite volume method (FVM) [17,20] based on the body-fitted coordinates is adopted. Fig. 2(a) shows a portion of a sample mesh for calculation; the mesh lines defines the boundaries of finite or control volumes (CVs). A typical CV is illustrated in Fig. 2(b). The idea of FVM is simply to integrate the governing equations over every CV. For example, for each CV, after the Gauss theorem is applied, a flux balance equation for the variables can be obtained with the following form:

$I_{e}-I_{w}+I_{n}-I_{s}+I_{t}-I_{b}+\int_{\Delta V} S_{\phi} \mathrm{d} V-\frac{\mathrm{d}}{\mathrm{d} \tau} \int_{\Delta V} \phi \mathrm{d} V=0$,

where $I_{i}, i=(e, w, n, s, t, b)$, represents the fluxes of variables across the faces of $\mathrm{CV}$ and $\phi=(u, v, w, P$ or $\theta)$; $\Delta V$ is the volume of $\mathrm{CV}$ and $S_{\phi}$ the source term of the governing equations. Each of the fluxes $I_{i}$ is made of two distinct parts, namely a convective contribution and a diffusive contribution; the mesh movement also needs to be considered in the convective part. Both terms are approximated with the central difference scheme; upwinding is found unnecessary here. More importantly,

(b)

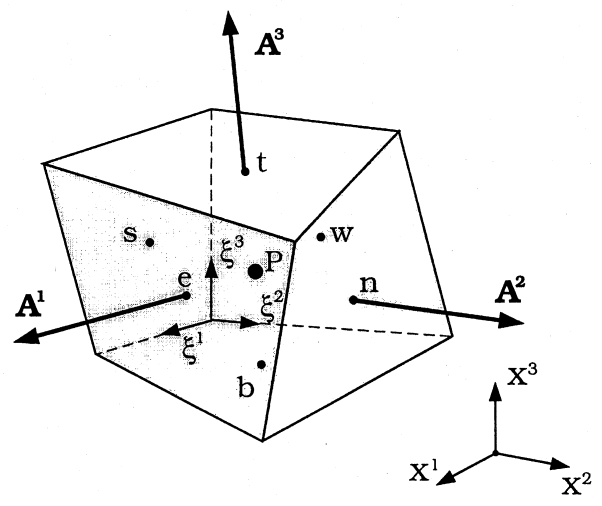

Fig. 2. (a) A portion of a sample mesh for calculation; (b) a typical finite volume. 
CVs with zero volume are also placed on boundaries. Accordingly, the boundary conditions can be imported into the flux balance equations easily. Detailed implementation of the scheme can be found elsewhere $[17,20]$. For stationary solutions, the time-dependent term is removed.

In order to locate multiple steady states for stationary solutions and to avoid the singular Jacobian matrix at bifurcation or limiting (turning) points, the pseudo arclength continuation [21] is further adopted. The idea of continuation is to trace the solution along a branch family through an arclength $s$ by including an additional equation for the continuation parameter $p$. For example, if $R a$ is chosen as the continuation parameter, the additional equation can be written as the following:

$$
\begin{aligned}
& \left.\frac{\partial \mathbf{x}^{\mathrm{T}}}{\partial s}\right|_{s_{0}} \cdot\left[\mathbf{x}(s)-\mathbf{x}\left(s_{0}\right)\right]+\left.\frac{\partial R a}{\partial s}\right|_{s_{0}}\left[R a(s)-R a\left(s_{0}\right)\right] \\
& \quad-\left(s-s_{0}\right)=0,
\end{aligned}
$$

where $\mathbf{x}=(u, v, w, P, \theta, h)^{\mathrm{T}}$ and $s-s_{0}$ is the step size along the branch, where the arclength $s \equiv\left\|(\mathbf{x}, R a)^{\mathrm{T}}\right\|_{2}$.

Appending the continuation equation to the steadystate flux balance equations (Eq. (11) without the transient term) for all CVs in the domain, as well as the isotherm condition (Eq. (9)), leads to a set of nonlinear algebraic equations:

$\mathbf{f}(\mathbf{y}) \equiv 0$,

where $\mathbf{y}=[\mathbf{x}, R a]^{\mathrm{T}}$. Eq. (13) is solved by Newton's method for all variables simultaneously. It should be pointed out that the beauty of Newton's method is that it solves all the variables globally and does not require any special interface update schemes for the unknown interface shape. During Newton's iterations, the Jacobian matrix $\tilde{J}$, defined as $\partial f_{i} / \partial y_{j}$, is estimated by the forward difference and the Newton's linear equations are solved by the GMRES method [22]. The preconditioner for GMRES used in this study is the incomplete $L U$ decomposition without fill in, i.e., the so called $I L U(0)$ preconditioner.

Although Newton's method provides a versatile solution to our problem, due to the limitation of computer memory (512 MB), the finest mesh we can use is quite limited. The total number of nonlinear algebraic equations is limited to $2 \times 10^{5}$. However, we have examined most of the standard solutions by using an iterative decoupled approach [20], and the numerical error using the mesh here is believed to be acceptable. Furthermore, although the leading eigenvalue of the Jacobian matrix from the Newton's method can be used for stability analysis [15], it is, unfortunately, still too costly to calculate for our 3D problem. Therefore, instead of using the eigenvalues, we have adopted a fully time-dependent calculation for the stability analysis. By providing a 3D disturbance, we can examine the time evolution of the responses. If the original state can be restored, the mode is stable. Otherwise, the solution is not stable. Furthermore, by monitoring the singularity of the Jacobian matrix through the test function $\tau_{i j}[15,23]$ during solution tracking, we are able to detect the bifurcation points.

\section{Results and discussion}

In the general formulation of this problem, the solutions depend on several parameters including $R a, \gamma$, $\operatorname{Pr}, A, L_{\mathrm{s}} / L_{\mathrm{m}}$, etc. However, in this study we focus mainly on the Prandtl number of one with the initial melt aspect ratio being one $(\operatorname{Pr}=1, A=1)$. For crystal growth or solidification applications, oxide materials have a comparable Prandtl number. The unity thermal conductivity ratio (i.e., $\kappa_{\mathrm{s}}=1$ ) is also reasonable. The effect of the solid amount $\left(L_{\mathrm{s}} / L_{\mathrm{m}}\right)$ will be discussed in the end. Although the parameters we consider here are very limited, we believe that the role of the interface for other situations may be similar.

Before calculated results are presented, several numerical tests on the numerical scheme have been performed to ensure the correctness of calculations. Fig. 3 shows the comparison of calculated thermal fields (an $m 1$ mode) with those obtained by Fluent [24], a commercial CFD package, for $L_{\mathrm{s}} / L_{\mathrm{m}}=0$ (with the interface being fixed) at $R a=4000 ; \Delta \theta=0.05$. As shown, good agreement is obtained for both the top-view isotherms at $x^{3}=0.5$ and the side-view ones at $x^{1}=0$. Other similar benchmark tests can be found elsewhere [25]. The effect of meshes on the $m 1$ family for the case with $5^{\circ}$ tilt is shown in Fig. 4; the interface is free and $L_{\mathrm{s}} / L_{\mathrm{m}}=1$. As shown, for $R a<5000$, the effect of meshes on the solution, for the mesh finer than $M 1$, seems to be insignificant. For stronger convection, a finer mesh may be necessary. However, in this study, the convection is generally very week $(R a \leqslant 8000)$. Therefore, we have
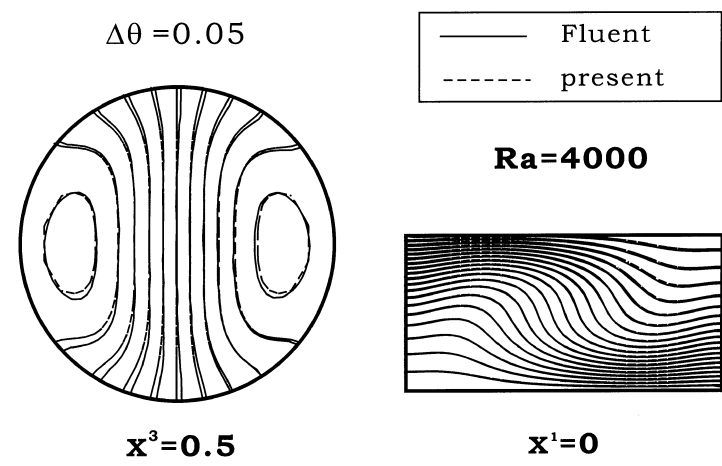

$\mathbf{R a}=\mathbf{4 0 0 0}$

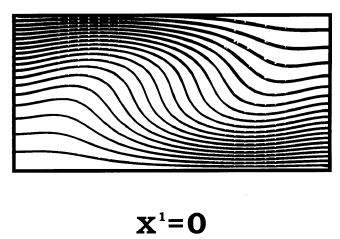

Fig. 3. Comparison of the calculated thermal fields of an $m 1$ mode at $R a=4000$ with those obtained by Fluent. 


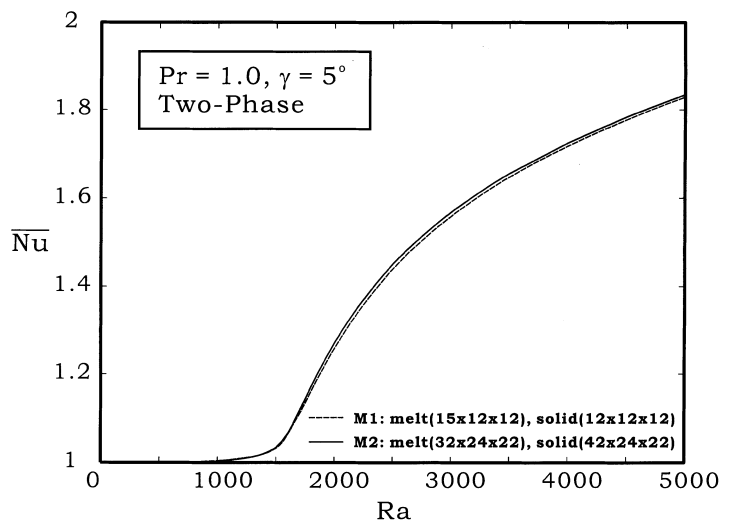

Fig. 4. The effects of meshes on $\overline{N u}$ for a $5^{\circ}$ tilt with a deformable interface.

chosen the mesh $M 2$ for key solution families ( $m 0$ and $m 1$ modes), while the mesh $M 1$ for the rest of solution branches. Further refinement of the solution is possible by using the segregated approach [20]. However, such an approach is difficult to obtain unstable branches. Furthermore, as mentioned previously, the stability of a solution family can be sensitive to the mesh resolution, and that is the main reason for the differences among some of the previous reports. In reality, due to the limitation of computing resources, it may be still difficult to obtain the so-called mesh independent bifurcation points for $3 \mathrm{D}$ problems. On the other hand, bifurcation structures are usually less sensitive to the numerical accuracy. Therefore, in this report, we do not attempt to settle the controversy among the previous studies for the fixed-interface problem. Instead, we will illustrate its key bifurcations, especially, when the deformable interface is introduced.

\subsection{Fixed interface $\left(A=1\right.$ and $\left.L_{\mathrm{s}} / L_{\mathrm{m}}=0\right)$}

As the interface is fixed and there is no-slip on the side wall, the bifurcation diagram for $\overline{N u}$ vs $R a$ is shown in Fig. 5(a); the unstable branches are indicated by dashed lines. Some typical solutions at $R a=8000$ are shown in Fig. 5(b). The solution structure for $2000<R a<4000$ is redrawn in Fig. 6, while the corresponding test function $\tau_{i j}$ along the static and $m 1$ branches is shown below. Some typical solutions are shown in Fig. 6 as well. In Figs. 5 and 6, the solution families with a clear symmetry at $x^{3}=0.5$ are marked by $m 0, m 1, m 2$, or $m 3$, respectively, for illustration. One can examine the solutions easily to get a better picture of the flow structures. As shown in Fig. 5(a) or Fig. 6, the first primary bifurcation is at $R a_{\mathrm{c}}=2250$ leading to an stable axisymmetric family $(m 0)$. This value is in good agreement with previous reported values (2250 by Hardin and Sani [12] and 2200 by Neumann [11]). The dramatic change of the test function along the static line is also in good agreement with the bifurcation point shown in Fig. 5(a) or Fig. 6. This bifurcation is also the onset of convection, which is a transition from the conductive to convective modes. The second and third primary bifurcations lead to $m 2\left(R a_{\mathrm{c}}=2480\right)$ and $m 1\left(R a_{\mathrm{c}}=2700\right)$ modes, respectively. Again, they are in reasonably good agreement with previous reported values as well $\left(R a_{\mathrm{c}}=2470,2700\right.$, respectively $\left.[12,14]\right)$.

Beside these primary bifurcations, however, the stability of the branching families is also a major interest in practice. However, it is still quite controversial.

(a)
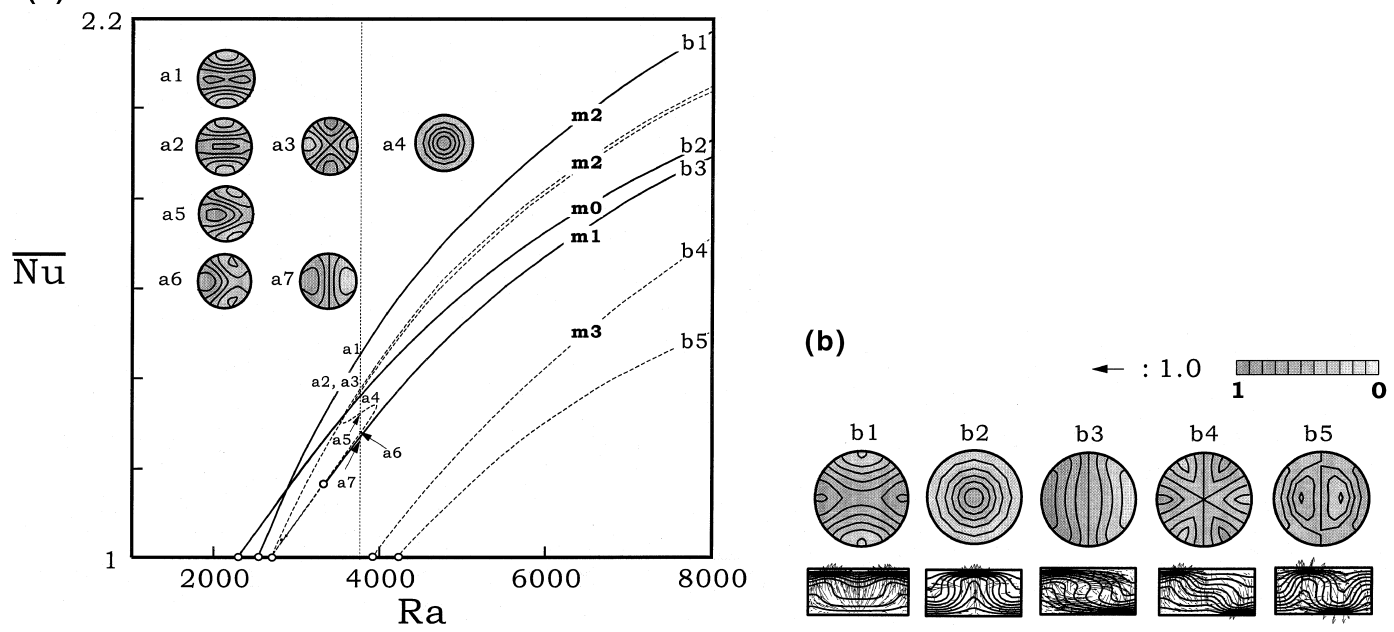

Fig. 5. Calculated results for the single-phase problem $\left(A=1\right.$ and $\left.L_{\mathrm{s}} / L_{\mathrm{m}}=0\right)$ : (a) bifurcation diagram; (b) some thermal and flow structures. 

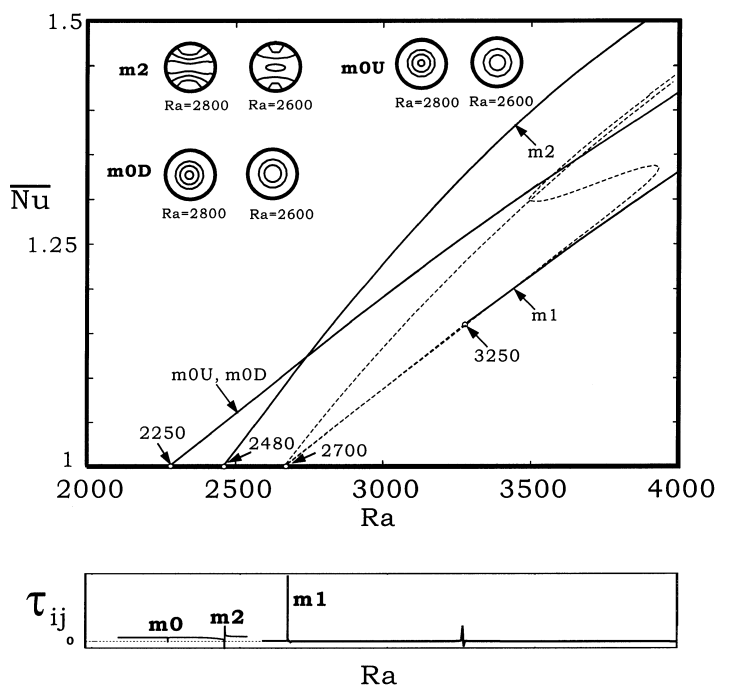

Fig. 6. A close-up view of Fig. 5 for $2000<R a<4000$ and the singularity test function $\tau_{i j}$ along the static and $m 1$ branches.

Wanschura et al. [14] tried to settle the arguments, but no conclusions have been made to date. They also obtained a stable $m 0$ solution up to about $5-10 R a_{\mathrm{c}}$ for $\operatorname{Pr}=6$.7. However, our $m 0$ solution becomes unstable at about $R a \approx 8000$, which can be identified by the change of the test function. We have also used the Fluent code to test the $m 0$ mode, and the stability limit is at about $R a=6000-7000$. Therefore, our stability limit for $m 0$ seems to be reasonable. However, if we use the mesh $M 1$ for calculation, the stability limit is much lower being at $R a \approx 3600$. As illustrated in Fig. 6, two stable $m 0$ modes are in the same branch due to the same $\overline{N u}$; one is marked by $m 0 U$ and one by $m 0 D$. The $m 0 U$ family has an upward flow at the centerline, while $m 0 D$ has a downward flow. These two solutions are stable for $R a<8000$ at least. Beyond this point, we are not able to get a stable solution. The difference between our results and previous ones is believed to be the mesh effect. However, even the $m 0$ mode can be stable up to higher $R a$, it is still very close to the neutral stability. As a result, it can become unstable easily as an imperfection is introduced. For lower $P r$ values, the $m 0$ mode becomes unstable at a much lower $R a$ value, and this can also be taken into account to our lower stability limit.

In Fig. 5(a) or Fig. 6, interestingly, the second primary bifurcation to the $m 2$ family is independent of the $m 0$ mode. The flow patterns at the intersection of the two branches are different; one is $m 0$ and one is $m 2$. The solution of the $m 2$ mode at $R a=8000$ is shown in the b1 of Fig. 5(b). From its isotherms at $x^{3}=0.5$, there are two hot spots (upward flows) and two cold spots (downward flows). The other $m 2$ solution at lower $R a$ is the solution a1 in Fig. 5(a). This solution branch is different from that proposed by Hardin and Sani [12] for $\operatorname{Pr}=6.7$. They showed that the intersection was a transcritical bifurcation for $P r=6.7$, where the $m 0$ and $m 2$ modes exchanged stability; the $m 0$ mode became unstable after this point, while the $m 2$ became stable. The $m 2$ mode branching from $R a_{\mathrm{c}}=2470$ seems to be quite stable. Its dynamic response for $R a=4000$ with respect to the tilt disturbance $\left(\gamma=0.5^{\circ} \sin (\pi t)\right.$ for $t<1$; $\gamma=0$ for $t>1$ ) is shown in Fig. 7(a). Interestingly, through mesh refinement, Wagner et al. [13] commented that for $P r=6.7$, the appearance of the $m 2$ mode (at $R a=2800)$ reported previously $[10,11]$ was due to the use of a too coarse grid, and the $m 2$ mode was not stable.

There are other types of $m 2$ modes, as shown in the solutions a 2 and a3 in Fig. 5(a), and they can also be $m 2 U$ and $m 2 D$ for the solution a2; similar finding for $\operatorname{Pr}=6.7$ was reported by Muller et al. [10]. Also, the solution a 3 is also a typical symmetric $m 2$ mode, and its symmetry-breaking bifurcation leads to the solution like a5. The solution a6 also bifurcates from the static line near $R a=2700$. However, these solutions are not stable and hence not so interesting. Furthermore, Wanschura et al. [14] also obtained the $m 2$ family for $P r=6.7$, but
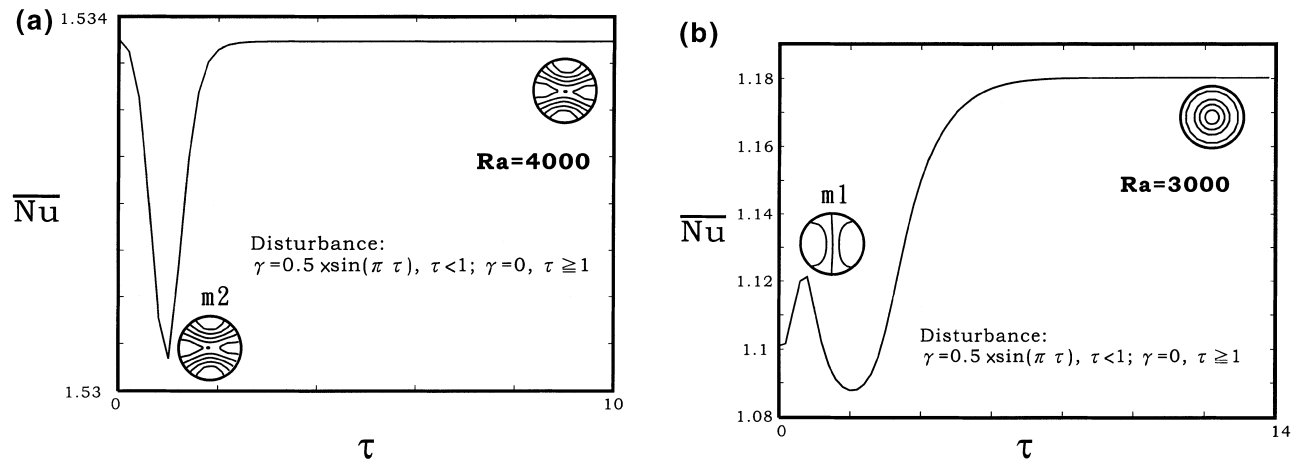

Fig. 7. Dynamic evolution of the basic state to an angular disturbance: (a) an $m 2$ mode at $R a=4000$; (b) an $m 1$ mode at $R a=3000$; the initial and final thermal fields are shown in the figures. 
they suspected that there was a small range that the $m 2$ mode may be unstable. From the geometric point of view, it is believed that a multi-cell family is favored for smaller aspect ratios. However, for $A=1$, it is not easy to judge a stable solution from such a consideration. As will be shown shortly, the $m 2$ modes are hard to survive after a $5^{\circ}$ tilt is introduced. Therefore, in practice, they may not be important as well.

The $m 1$ mode is obtained from the third primary bifurcation point in Fig. 5(a) or Fig. 6 is found unstable until $R a=3250$. Wanschura et al. [14] obtained the neutral value at about $R a>4224$. We are able to get a stable $m 1$ solution at $R a=4000$ by both our and Fluent codes. The comparison of the $m 1$ solutions has been shown in Fig. 3. The solution connectivity at the secondary bifurcation is still unknown. The time-dependent evolution of the $m 1$ mode at $R a=3000$ after an angular disturbance is shown in Fig. 7(b). As shown, the solution runs away, and finally becomes an $m 0$ mode. Clearly, as shown in Fig. 6, four stable solutions may exist after $R a=3250$. At smaller $R a$, the solutions $m 0$ and $m 2$ seem to be more stable.

Other modes, such as the $m 3$ family like solutions b4 and b5 in Fig. 5(b), are found. Again, these modes are unstable and thus not much interesting in practice.

In addition to using the pulse disturbance, the stability of a solution family can be further investigated though symmetry breaking. In practice, the system may not be perfectly aligned with the gravity. Therefore, a little tilt against the gravity is a typical symmetry breaking that leads to imperfect bifurcations. In general, less stable modes can be removed or become unstable, and as a result, the bifurcation structures become sim- pler and the multiplicity decreases. As shown in Fig. 8 (a), at $5^{\circ}$ tilt, the $m 0$ and $m 2$ modes disappear from our bifurcation diagram. At least, we could not obtain their family anymore by using the solution at $\gamma=0$ as an initial guess. Indeed, as discussed by Lan et al. [15], the tilt reduces multiplicity and results in a much simpler solution structure. As shown in Fig. 8 (a), the primary mode now becomes $m 1$, which starts from $R a=0$. Typical isotherms and flow patterns are al and b1 in Fig. 8(b). In this branch, the flow direction follows the tilt, as shown by the solution a1. On the other hand, the other flow direction against the tilt, such as in the solution b2, is also stable. Without tilt, the solutions b1 and $\mathrm{b} 2$ are images to each other; physically, they are the same. Hence, the tilt breaks the symmetry. The reason for $\mathrm{b} 2$ being stable is quite simple. As a flow like b2 is developed, the small tilt against the flow is not able to reverse it due to the inertial effect. However, at weaker convection below the turning point, the inertial term is not large enough to against the tilt, and as a result, only the b1 family exists.

Tracking the solution family downward from b2, we obtain an unstable family (b3) through a turning point. Such an imperfect bifurcation is generic, which is in general obtained from a pitchfork bifurcation. Similar structures were found in the 2D problem [15]. The other imperfect $m 3$ modes was also obtained, such as the families for b4 or b5, but they are still unstable.

By comparing Figs. 5 and 8 , it is clear that the $m 1$ family seems to be more stable for $A=1$, while the axisymmetric $m 0$ modes and $m 2$ modes are less stable with respect to the symmetry breaking. Nevertheless, multiplicity still exists, and the final stable mode is indeed
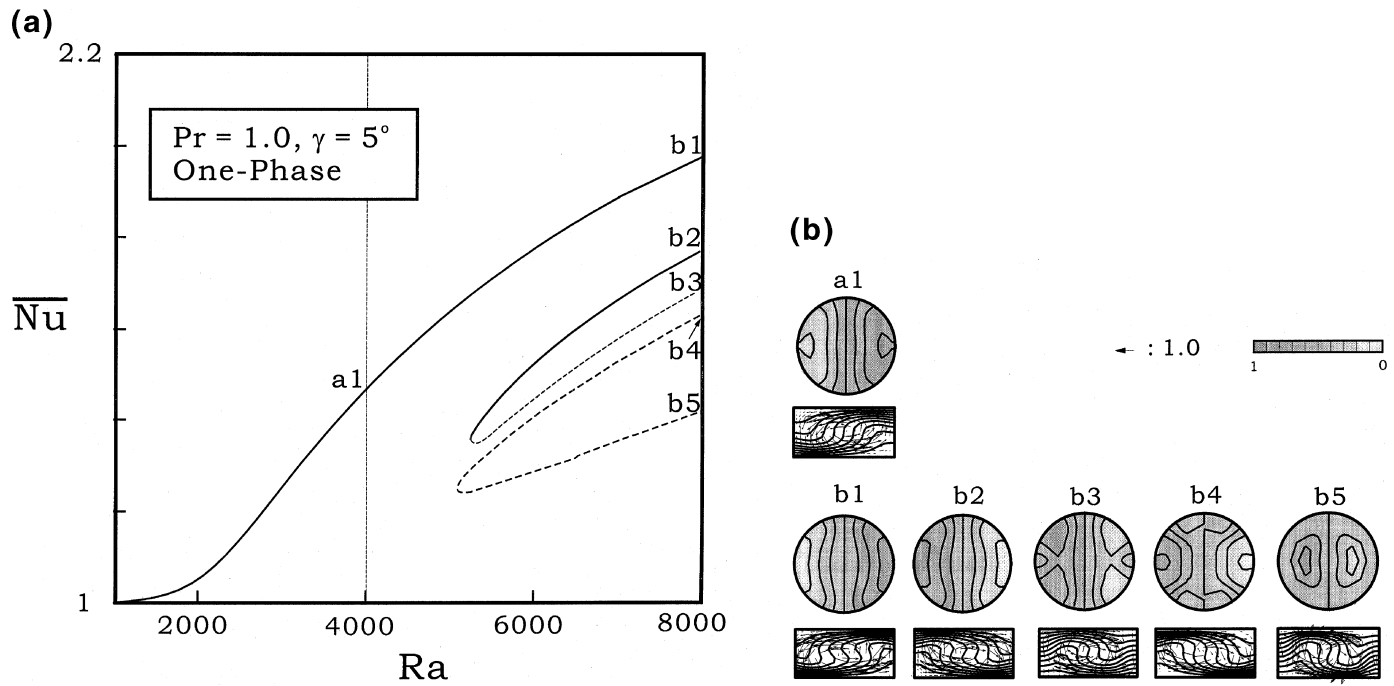

Fig. 8. Calculated results for the single-phase problem $\left(L_{\mathrm{s}} / L_{\mathrm{m}}=0\right)$ with a $5^{\circ}$ tilt: (a) bifurcation diagram; (b) some thermal and flow structures. 
path dependent. Furthermore, the branching families with even modes, such as $m 0$ or $m 2$, become less stable when an asymmetric condition is introduced unless there are other stabilizing mechanisms involved. As will be illustrated shortly, the interface deformation may form a barrier to inhibit the $m 0$ mode from running away.

\subsection{Deformable interface $\left(A=1\right.$ and $\left.L_{\mathrm{s}} / L_{\mathrm{m}}=1\right)$}

As the interface is allowed to deform, the bifurcation structure changes significantly. We take a basic conduction mode with the same amount of solid and melt, i.e., $L_{\mathrm{s}} / L_{\mathrm{m}}=1$, as an example for illustration; the heat flux from the bottom is kept the same as before, so that $\overline{N u}=1$. The bifurcation diagram is shown in Fig. 9(a) and some typical thermal and flow fields are shown in Fig. 9(b). As shown, the onset mode is no longer $m 0$, but $m 1$ and the bifurcation becomes subcritical. The primary bifurcation point $\left(R a_{\mathrm{c}}=2130\right)$ is also significantly lower than the fixed one ( 2250 for $m 0$ and 2700 for $m 1$ ), even though the zone length and the aspect ratio at the onset point are exactly the same as before. The $m 1$ family bifurcating from the static mode is unstable first (dashedline), but becomes stable after the turning point $(R a=2000)$. Typical stable $m 1$ solutions like $\mathrm{a} 1, \mathrm{~b} 1$, and c2 are shown in the first column of Fig. 9(b). As shown, as the flow develops, the interface shape is shaped by the convection significantly. The melt height increases sig- nificantly as well. As a result the solutions move away from the fixed-interface ones. However, as the melt aspect ratio $A$ increases, the $m 1$ mode is preferred. The codimension-two point for the fixed-interface problem is at about $A=1.12$ and $R a=2700$ [14], where the $m 0$ and $m 1$ modes may appear at the same time. Therefore, away from the static line, due to the larger melt height, the $m 1$ mode predominates.

At the static line, the subcritical nature is due to the coupling of the flow and the interface. Davis et al. [18] first reported such a phenomena for a thin layer melted from below, and the transition to a hexagon mode was subcritical, i.e., with a jump in the melt-layer thickness. Good agreement between experiments and their weakly nonlinear analysis was obtained. They also found that the subcritical nature predominated for a thick solid layer. We have also obtained a similar conclusion that the subcriticality becomes obvious as the solid amount increases, which will be illustrated shortly. Furthermore, once the $m 1$ mode has been developed, it will need a much lower $R a$ (below the turning point) to get back to its conductive state. The decrease of the heat transfer rate at the turning point is also dramatic. For a thick solid layer, this quenching point can even be lower than 1708 , i.e., the classic onset point.

Beside the first appearing mode, the second primary bifurcation is to an axisymmetric $m 0$ mode, and again, it is subcritical (not very clear). However, due to the de- (a)

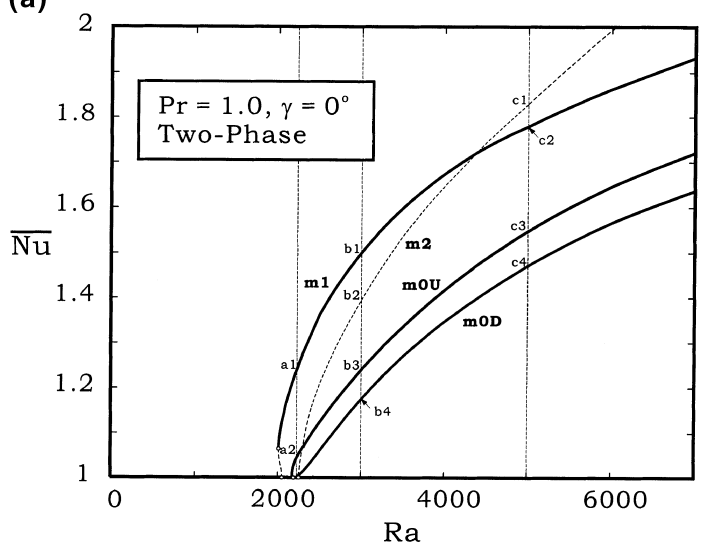

(b) a 1
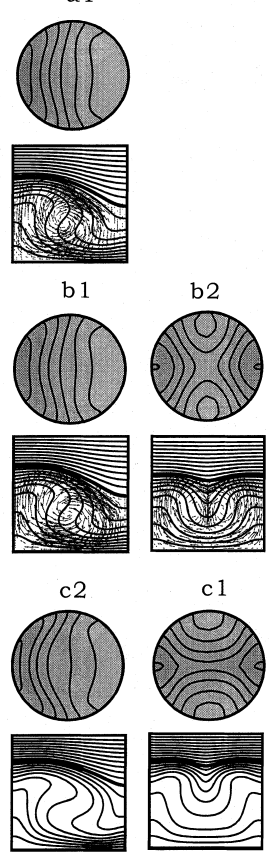
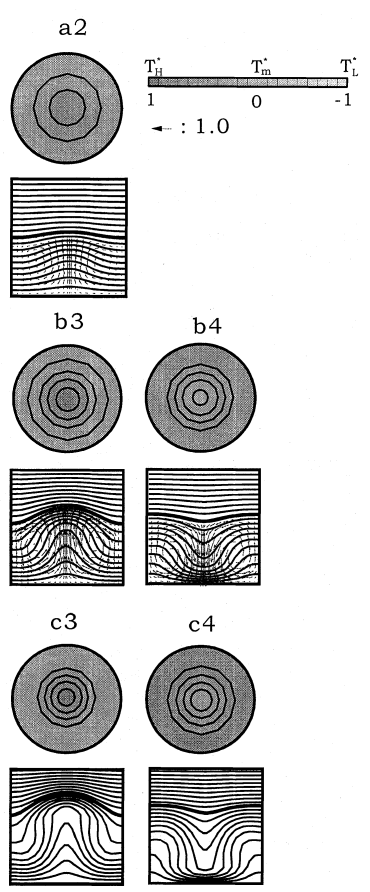

Fig. 9. Calculated results for the two-phase problem $\left(A=1\right.$ and $\left.L_{\mathrm{s}} / L_{\mathrm{m}}=1\right)$ : (a) bifurcation diagram; (b) some thermal and flow structures. 
formation of the interface caused by the convection, the $m 0 D$ and $m 0 U$ modes do not have the same $\overline{N u}$, i.e., having different heat transfer rates across the interface. For the upper branch, it soon becomes stable after the turning point. As shown in the solutions a2, b3, or c3 of Fig. 9(b) (the third column), this mode is $m 0 U$; the melt flows upward at the center. Due to the melt convection, the interface becomes concave. On the other hand, the lower branch has a different flow direction, i.e., $m 0 D$. As shown in the fourth column of Fig. 9(b), the typical solutions are $\mathrm{b} 4$ and $\mathrm{c} 4$, and their interfaces are convex. Indeed, right at the static line, there is a transition from the $m 0 D$ to $m 0 U$ modes, where the interface concavity also changes. To better illustrate the bifurcation, we represent the bifurcation diagram using a new $\overline{N u}$, denoted by $\overline{N u}^{*}$, by subtracting the original $\overline{N u}$ by one (the static mode) and adding a minus sign to it for the $m 0 D$ mode. As shown in Fig. 10, near the static line, it is clearly a transcritical bifurcation. Beside the dash-line, which is an $m 0 U$ mode, both $m 0 D$ and $m 0 U$ families are stable. Similar transcritical bifurcation was also observed in the 2D case (a rectangular system) [15], and the bifurcation point was also due to the change of interface concavity; the turning point also appear in the concaveinterface branch. Furthermore, as compared with the $m 0$ mode in Fig. 6, the $m 0$ modes here is more stable, even using the mesh $M 1$. The interface shape, on the other hand, may provides a stabilizing mechanism that confines the $m 0$ flow and hinders it from running away. This will become obvious as the $5^{\circ}$ tilt is introduced, which will be discussed shortly.

As illustrated in Fig. 9(a), an $m 2$ mode appears lately behind $m 0 D$, but it is unstable; it is stable for the fixedinterface case. This is also an indication that the pres-

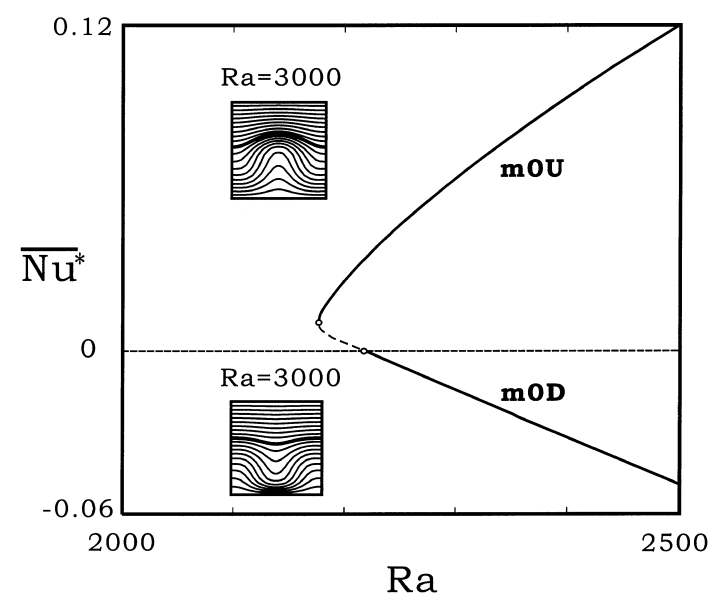

Fig. 10. A redrawn transcritical bifurcation diagram from Fig. 9(a) for the $m 0$ modes; $\overline{N u}^{*}$ is defined by subtracting $\overline{N u}$ by one and adding a minus for the $m 0 U$ mode. Two solutions at $R a=3000$ are shown. ence of the deformable interface may destabilize the system, easing the onset of the convection. One can also imagine that the interface morphology of the $m 2$ mode is more complicated, and this may discourage its formation. Also, the increasing melt aspect ratio due to convection does not favor the $m 2$ family. Typical solutions are shown in the second column of Fig. 9(b) like solutions $\mathrm{b} 2$ and $\mathrm{c} 1$. Other $m 2$ or higher-order modes are also found but they are not stable and thus not interesting. Therefore, we do not attempt to go any further. Furthermore, even though the $m 2$ mode is unstable for $R a>2300$, we still have three stable modes, i.e., $m 1$, $m 0 U$, and $m 0 D$. In reality, which mode being selected is indeed path dependent. Interestingly, these solutions are, at least, stable up to $R a=8000$. The multiplicity exists over such a wide range is also different from that with a rigid interface. Again, it believed that the interface shape confines the flows and retain them to a higher $R a$ value. As the $R a$ number increases, the interface deformation increases as well, and this further inhibits the flow bifurcation. The stability of these modes can be further illustrated through imperfect bifurcations.

An imperfect bifurcation due to $5^{\circ}$ tilting is shown in Fig. 11(a). This bifurcation diagram is similar to the fixed-interface one (Fig. 8). The subcriticality also disappears; it exists within a very small tilt angle. Again, the most stable family is still $m 1$ branching from $R a=0$, and it has a flow following the tilt direction. Typical solutions from this branch are illustrated by the solutions a1, b1, and $\mathrm{cl}$ in Fig. 11(b). Other two $m 1$ modes are related to the solutions b2 (or c2) and b3 (or c3), respectively. Again, similar to the fixed-interface ones, one is stable and one is not.

Interestingly, an imperfect $m 0$ mode is still retained, and its upper branch is stable, such as the solutions b4 and c4. As shown in the fourth column of Fig. 11(b), this stable branch is an imperfect $m 0 U$ mode. The lower branch, which is believed to be related to the $m 0 D$ mode, is unstable. Typical solutions of the unstable imperfect $m 0 D$ mode are illustrated in the solutions b5 and c5 of Fig. 11(b). Again, one may compare Figs. 9(a) and 11(a) and find that the $m 0$ family is detached from the static line after the $5^{\circ}$ tilting because its perfect mode does not exist. This solution structure here is also somewhat different from that of the 2D case [15], where two pairs of convex and concave solutions appear after tilt. Furthermore, even with $5^{\circ}$ tilt, we still have three stable modes after the turning point $(R a \approx 2800)$ of the $m 1$ mode.

As mentioned previously, the subcriticality of the primary bifurcation is affected by the solid amount. To further illustrate that, we take the thickness ratio $L_{\mathrm{s}} / L_{\mathrm{m}}$ as a parameter to illustrate the primary bifurcation. The heat flux from the bottom is kept the same by changing the upper thermal boundary condition. As shown in Fig. 12(a), with the increasing solid layer thickness (or solid 

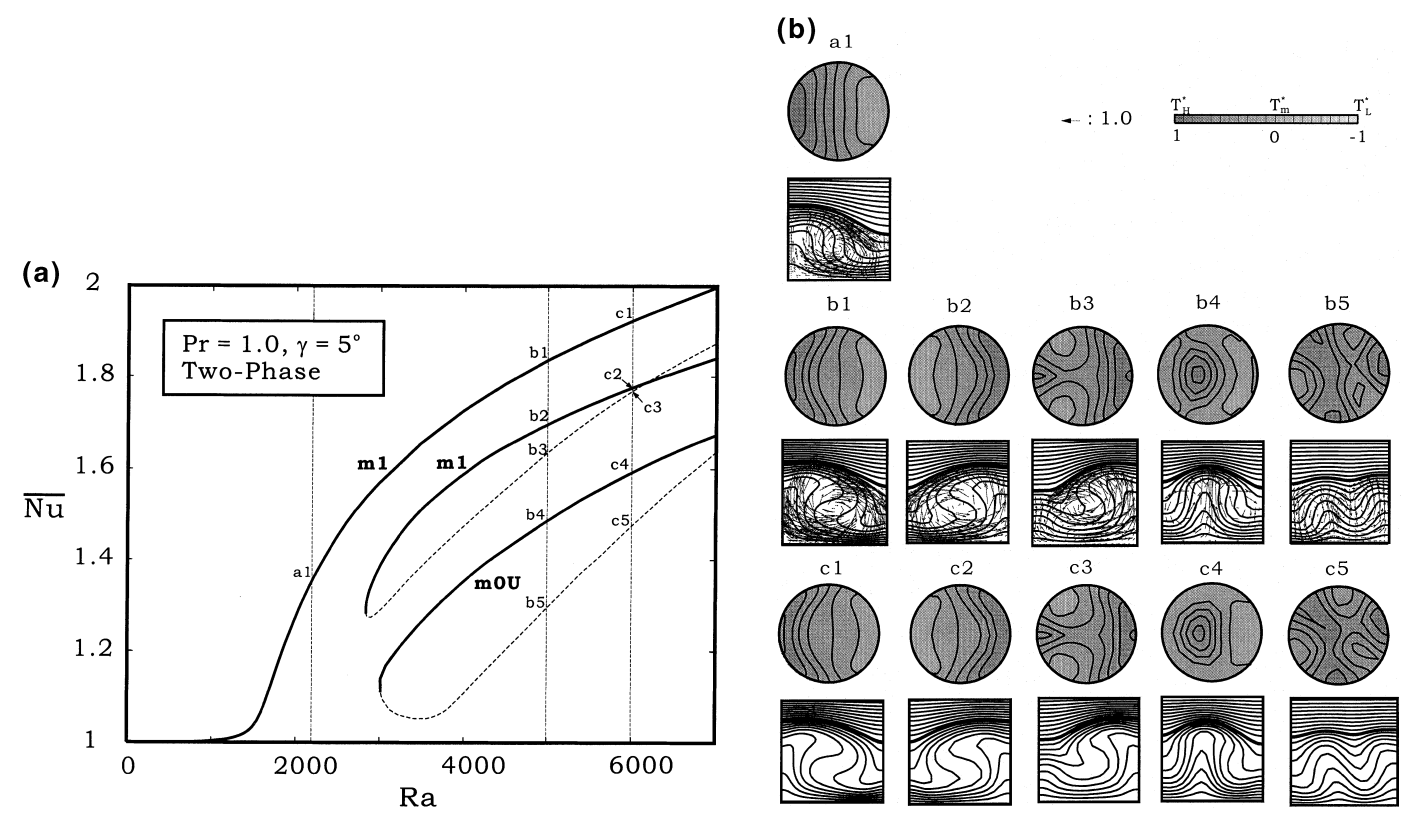

Fig. 11. Calculated results for the two-phase problem with a $5^{\circ}$ tilt: (a) bifurcation diagram; (b) some thermal and flow structures.
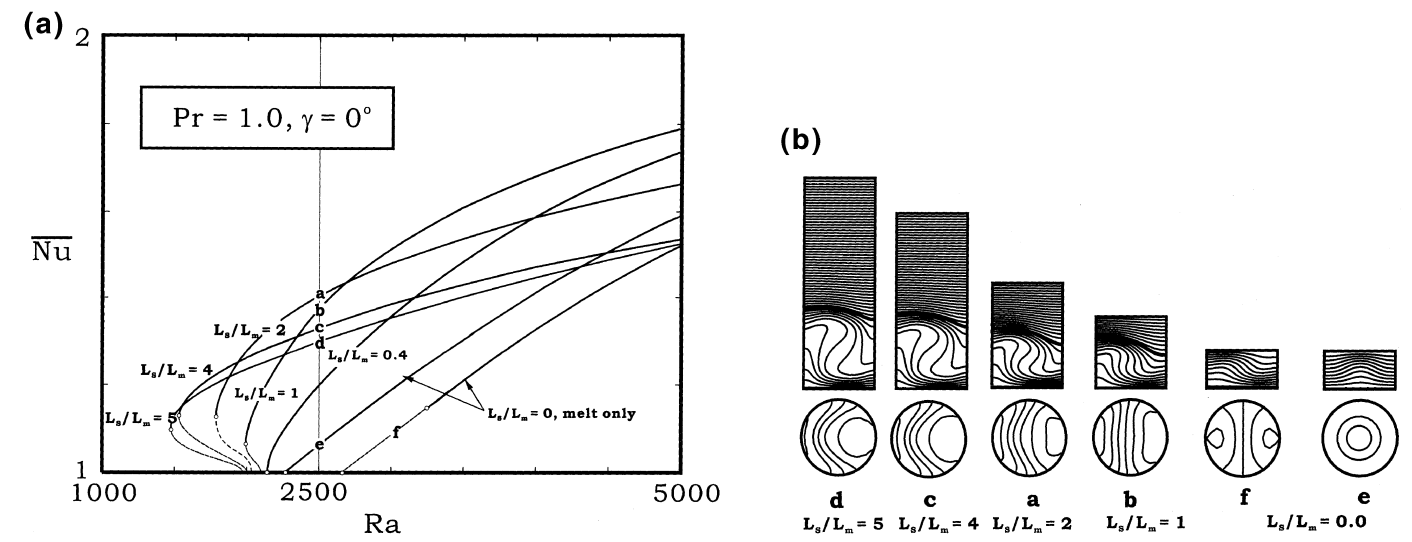

Fig. 12. The effect of solid amount on the primary bifurcation: (a) bifurcation diagram; (b) thermal fields and interface shapes at $R a=2500$. The heat flux for all cases is kept the same.

amount), the bifurcation of the $m 1$ mode changes indeed from supercritical to subcritical. Solutions at $R a=2500$ are also shown in Fig. 12(b); the $m 0$ mode is also included for comparison. As shown, when the solid layer thickness is zero, which can be done numerically, the solution is the same as the fixed-interface one. However, there is a dramatic change of the solution when the solid layer becomes finite. The subcriticality occurs at about $L_{\mathrm{s}} / L_{\mathrm{m}} \approx 0.5$. Further increasing the solid amount, both the primary and turning points move forward (to the left). However, for $L_{\mathrm{s}} / L_{\mathrm{m}}>4$, the change becomes much smaller. This result is also consistent with the weakly nonlinear analysis for a thin-layer of material melted from below in an infinite domain by Davis et al. [18] that the subcriticality to hexagon cells predominates for a thick solid layer.

On the other hand, the effect of the solid layer has little effect on the bifurcation point of the $m 0$ mode. Therefore, as the solid layer increases, the primary bifurcation point of the $m 1$ mode moves forward, and to a certain point, at about $L_{\mathrm{s}} / L_{\mathrm{m}}=0.3$, both modes appear at the same time, i.e., a co-dimension-two point. At this point, the onset of the convection can be $m 0$ or $m 1$ depending on the initial disturbance. Furthermore, the subcriticality nature is particularly interesting in practice. The onset of convection occurs dramatically to a 
finite convective mode as the subcritical point is passed. On the other hand, a finite convective mode can be quenched to a conductive mode by reducing the driving force to be below the turning point. Such a hystersis may cause a process difficult to control or operate.

\section{Conclusions}

In this study, we have explored the 3D nonlinear phenomena of a generic two-phase Rayleigh-Benard problem in a cylinder. The fully nonlinear analysis is based upon a finite-volume/Newton's method with a solution tracking capability. A singularity test function and dynamic calculations have also been used to locate the bifurcation points and to examine the stability of solution families. The bifurcation diagrams for $\operatorname{Pr}=1$ and $A=1$ have been constructed for both fixed and free interfaces.

It is found that the solid layer plays a critical role in the two-phase problem, both on the flow onset and the convection modes. As the solid layer diminishes to zero, the free-interface solution is the same as the fixed-interface one. For the fixed-interface problem, the first primary bifurcation is either $m 0 U$ or $m 0 D$, the second appearing mode is an $m 2$ mode, and then is an $m 1$ mode. Other unstable or high-order bifurcations are also found, but they seem to be unimportant in practice and are vulnerable to imperfections. As the solid amount increases, the onset of the $m 1$ mode moves forward $\left(R a_{\mathrm{c}}\right.$ is reduced), while the onset points of the $m 0$ and $m 2$ modes are not changed much. As a result up to a certain solid amount, the first appearing mode becomes $m 1$. As the solid amount is further increased, the primary bifurcation becomes subcritical. Due to the confinement of the interface shape, the $m 0$ modes becomes more stable, and its transcritical bifurcation is similar to the $2 \mathrm{D}$ one [15], where the transcritical point marks the change of interface concavity. Furthermore, with the interface deformation, as compared with a fixed-interface one, the $m 0 U$ mode is also more stable and can be retained even with a $5^{\circ}$ tilt.

Although the solution structures obtained here are for $P r=1$, according to the 2D results [15], we do not expect much change along the static line for different $\mathrm{Pr}$ numbers. However, away from the static line, the effects are not trivial. Nevertheless, the bifurcation structures presented here are quite generic, which may provide useful information for further study.

We have also found multiple stable steady states for both fixed and free-interface problems and the illustrated solution structures also provide the basis for further analysis. The imperfect bifurcations due to $5^{\circ}$ tilt also help further identify the stability of a solution family. As mentioned, those modes near the neutral stability, which may be quite controversial from different calculations, can be destroyed easily. Again, the modes that survive under the imperfection are more stable, and may be easier to be observed in experiments. Furthermore, in practice, all the stable modes are path dependent, and are sensitive to the initial conditions and disturbances. Nevertheless, with the solution structure in mind, it may be easier to better control the system. In addition, other parameters, such as the thermal conductivity ratio of the melt and the solid phases and the effects of boundary conditions, etc., may be important as well, but they are beyond the scope of this study.

On the other hand for 3D problems, numerical accuracy is indeed affected by grid resolution. However, the computation effort increases significantly as the mesh is refined. Therefore, even for the fixed-interface problem, there are still some disagreements on the solution stability and structures in the literature, particularly, for the bifurcation points with a higher null dimension. On the other hand, because these high-order bifurcations are usually structurely unstable, they are difficult to find experimentally. In other words, these bifurcation may not be so interesting, even though they are helpful in constructing detailed diagrams. Furthermore, they are, unfortunately, usually very sensitive to the mesh and hard to calculate accurately. On the contrary, imperfect structures are more generic and structurely stable and can be easily obtained both experimentally and numerically with a much better confidence. Therefore, the tilt introduced here also help map the key picture of the nonlinear bifurcations.

Finally, solutal effects [26,27] and time-dependent modes [14] are not considered in this work. It is believed that similar solution structures due to the solutal effects may be found. Furthermore, investigating the 3D timedependent modes, which are believed to occur at higher $R a$ numbers, still remains a grand challenge for numerical simulation. Again, the grid resolution is a major concern. The control of the bifurcation through rotation or other external forces like magnetic fields may be interesting as well, and they will be discussed in the near future.

\section{Acknowledgements}

This work is supported by the National Science Council of the Republic of China under Grant No. NSC 85-2214-E-008-014.

\section{References}

[1] J. Pannu, G. Joglekar, P.A. Rice, Natural convection to cylinders of phase change material used for thermal storage, AIChE Symposium Series (1980) 47-55. 
[2] H.W. Hale, R. Viskanta, Solid-liquid phase change heat transfer and interface motion in materials cooled from above and below, Int. J. Heat Mass Transfer 23 (1980) 283-292.

[3] G. Muller, A. Ostrogorsky, Convection in melt growth, in: D.T.J. Hurle (Ed.), Handbook of Crystal Growth, NorthHolland, Amsterdam, 1993, pp. 709-819.

[4] D.T.J. Hurle, E. Jakeman, Introduction to the techniques of crystal growth, PhysicoChem. Hydrodyn. 2 (1981) 237244.

[5] D.T.J. Hurle, E. Jakeman, A.A. Wheeler, Hydrodynamic stability of the melt during solidification of a binary alloy, Phys. Fluids 23 (1983) 624-627.

[6] S.F. Liang, A. Vidal, A. Acrivos, Buoyancy-driven convection in cylinder geometries, J. Fluid Mech. 86 (1969) 239-256.

[7] G.S. Charlson, R.L. Sani, Thermoconvective instability in a bounded cylindrical fluid layer, Int. J. Heat Mass Transfer 13 (1970) 1479-1496.

[8] G.S. Charlson, R.L. Sani, Finite amplitude axisymmetric thermoconvective flow in a bounded cylindrical layer of fluid, Int. J. Heat Mass Transfer 14 (1971) 2157-2160.

[9] Y. Yamaguchi, C.J. Chang, R.A. Rrown, Multiple buoyancy-driven flows in vertical cylinder heated from below, Philos. Trans. R. Soc. Lond. A 312 (1984) 519-552.

[10] G. Muller, G. Neumann, W. Weber, Natural convection in vertical Bridgman configurations, J. Crystal Growth 70 (1984) 78-93.

[11] G. Neumann, Three-dimensional numerical simulation of buoyancy-driven convection in vertical cylinders heated from below, J. Fluid Mech. 214 (1990) 559-678.

[12] G.R. Hardin, R.L. Sani, Buoyancy-driven instability in a vertical cylinder: binary fluids with Soret effect. Part II: weakly non-linear solutions, Int. J. Numer. Methods Fluids 17 (1993) 755-786.

[13] C. Wagner, R.C. Friedrich, R. Narayanan, Comments on the numerical investigation of Rayleigh and Marangoni convection in a cylinder, Phys. Fluids 6 (1994) 1425-1433.

[14] M. Wanschura, H.C. Kuhlmann, H.J. Rath, Three-dimensional instability of axisymmetric buoyant convection in cylinders heated from below, J. Fluid Mech. 326 (1996) 399-415.

[15] C.W. Lan, M.C. Liang, M.K. Chen, Stability and bifurcation analyses of a two-phase Rayleigh Benard problem in a cavity, Phys. Fluids 10 (1998) 1329-1343.

[16] C.J. Chang, R.A. Brown, Natural convection in steady solidification: finite element analysis of a two-phase Rayleigh-Benard problem, J. Comput. Phys. 53 (1984) 1-26.

[17] M.C. Liang, C.W. Lan, A finite-volume/Newton method for a two-phase flow problem using primitive variables and collocated grids, J. Comput. Phys. 127 (1996) 330-345.

[18] S.H. Davis, U. Muller, C. Dietsche, Pattern selection in single-component systems coupling Benard convection and solidification, J. Fluid Mech. 144 (1984) 133-151.

[19] L.D. Landau, E.M. Lifshitz, Fluid Mechanics, second ed., vol. 6, Pergamon Press, Elmsford, 1987, p. 217.

[20] C.W. Lan, M.C. Liang, Multigrid methods for incompressible heat flow problems with an unknown interface, J. Comput. Phys. 152 (1999) 55-77.

[21] H.B. Keller, Numerical solution of bifurcation and nonlinear eigenvalue problems, in: P.H. Rabinowi (Ed.), Applications of Bifurcation Theory, Academic Press, New York, 1977, pp. 359-384.

[22] Y. Saad, M.H. Schultz, GMRES: a generalized minimal residual algorithm for solving nonsymmetric linear systems, SIAM J. Sci. Stat. Comput. 7 (1986) 856-869.

[23] R. Seydel, Numerical computation of branch points in nonlinear equations, Numer. Math. 33 (1979) 339-352.

[24] Fluent UNS 5.0 User Manual, Fluent Inc., 1999.

[25] C.W. Lan, M.C. Liang, Three-dimensional simulation of vertical zone-melting crystal growth: from symmetry breaking to multiple states, J. Crystal Growth 208 (2000) 327-340

[26] G.B. McFadden, S.R. Coriell, Solutal convection during directional solidification, in: Proceedings of the First National Fluid Dynamics Congress, Cincinnati, OH, 2528 July 1988.

[27] M.D. Impey, D.S. Riley, A.A. Wheeler, K.H. Winters, Bifurcation analysis of solutal convection during directional solidification, Phys. Fluids A 3 (1991) 535-550. 